\title{
Coulisses
}

Revue de théâtre

\section{La facette de la paradidascalie}

Sur la « didascalie » intégrée au dialogue

\section{Witold Wolowski}

\section{OpenEdition}

Journals

Édition électronique

URL : https://journals.openedition.org/coulisses/1467

DOI : $10.4000 /$ coulisses. 1467

ISSN : 2546-9460

Éditeur

Presses universitaires de Franche-Comté

\section{Édition imprimée}

Date de publication : 31 décembre 2009

Pagination : 131-144

ISBN : 978-2-84867-270-0

ISSN : $1150-594 \mathrm{X}$

Référence électronique

Witold Wolowski, «La facette de la paradidascalie », Coulisses [En ligne], 39 | Automne 2009, mis en ligne le 30 novembre 2016, consulté le 29 décembre 2022. URL : http://journals.openedition.org/ coulisses/1467; DOI : https://doi.org/10.4000/coulisses.1467

Ce document a été généré automatiquement le 29 décembre 2022.

Tous droits réservés 


\title{
La facette de la paradidascalie
}

Sur la « didascalie » intégrée au dialogue

\author{
Witold Wolowski
}

\section{La bicanalité trangressée ou le retour à l'unicanalité originelle?}

1 Si la représentation théâtrale est presque toujours sémiotiquement pluricode (Klinkenberg 2000: 232), le texte de théâtre est d'habitude bicanal. Je prends la précaution d'utiliser presque et d'habitude pour ne pas exclure du domaine théâtral des cas extrêmes de l'écriture dramatique, à savoir des spectacles où la didascalie " a entièrement phagocité le dialogue » (Thomasseau 1998 : 132) et des ouvrages dialogaux sans stratification énonciative (dialogues sans marquage de la DSL ${ }^{1}$ ) ou sans zonage (Wołowski 2007b : $329 \mathrm{ss}$ ). La bicanalité du texte dramatique ou, en termes ingardeniens, le principe de la double projection des états de choses (Ingarden 1931: 209-210) ${ }^{2}$, serait ainsi ce sur quoi se fonde la singularité de l'écriture théâtrale en tant que genre de discours. Le texte de théâtre est un texte dialogo-didascalique, sinon il est légitime de le reléguer aux confins de sa propre catégorie générique ; tel est du moins le point de vue qu'il semble censé d'adopter, si l'on tient à préserver une conception relativement nette de la théâtralité textuelle.

2 Le dramaturge dispose ainsi de deux " pinceaux " pour écrire sa pièce, y inscrire une mise en scène virtuelle et prédéfinir, au moins partiellement, le rapport entre les différents codes intervenant dans l'éventuelle création concrète. Or, dès qu'il y a deux pinceaux, la tentation de les mêler ne tarde pas à se faire pressante. En fait, les auteurs dramatiques ont compris, dès la moitié du XIX siècle, qu'ils peuvent faire des deux canaux énonciatifs du texte de théâtre un objet d'expérimentations artistiques et non pas seulement une norme à sauvegarder.

3 Il importe pourtant d'observer que cette tentation n'en est pas vraiment une, dans la mesure où la bicanalité, telle que nous la connaissons aujourd'hui, est elle-même une convention qui ne s'affirme qu'avec le xviII ${ }^{\mathrm{e}}$ siècle et la "révolution opérée par le drame bourgeois », comme le note G. Zaragoza (2007: 87). La didascalie interne, i.e. dite 
sur la scène par un agent destiné à l'oraliser ou diluée dans l'échange dialogal des dramatis personae, a été, dès le début du théâtre, un état de fait naturel, même si - et cela demeure crucial - le théâtre avait, dès l'origine, ménagé un espace particulier aux instances annonciatrices, commentatrices et coordinatrices, tels le coryphée et le chœur - véritables didascalies parlantes. Une brève citation :

LE CORYPHEE. Eh bien, Perses, allons! Installons-nous sous cet antique toit; réflechissons [...] quel peut être le sort de Xerxès, notre roi [...]. Mais voici venir dans sa gloire - la prunelle des dieux n'est pas plus éclatante - la mère du grand roi : ma reine. A son approche je tombe sur la face. Et nous lui devons tous marquer par nos paroles hommage et bienvenue. (Eschyle, Les Perses, in: Les Tragiques grecs, p. 34).

4 Tout est là, mixé dans un discours pluriel d'une instance unique: $1:$ une trace d'échange dialogal sous forme d'apostrophe adressée aux autres personnages en scène ; $2:$ une réflexion méta-diégétique; $3:$ une paradidascalie proscénique annonçant l'entrée en scène d'un personnage nouveau ; $4:$ une paradidascalie diégétique de type descriptif (très poétique, soit-dit en passant) ; 5 : une injonction adressée aux autres participants de la scène. Dans cet extrait, dialogue et didascalie ne font donc qu'un, et on pourrait donner des centaines d'exemples semblables dans le corpus des tragiques grecs. Si l'on veut ainsi porter un jugement équilibré sur la caducité du clivage binaire entre la didascalie et le dialogue, comme l'avait dit M. Bernanoce (2007: 49), il faut avoir toujours présent à l'esprit le fait que bon nombre de nos observations actuelles à propos des expansions, infractions et excès de la didascalie ne sont au fond qu'un effet du phénomène de la rétro-projection générique. Le siècle des Lumières avait désuni l'unité quasi-organique du texte de théâtre et nous affirmons aujourd'hui que le retour à l'union fait figure de procédé, de technique, de stratégie.

\section{La paradidascalie (PDI)}

5 Le récent engouement pour la didascalie - depuis les travaux de M. Issacharoff (1985) jusqu'au projet international de F. Fix et F. ToudoireSurlapierre (2007) - a eu pour effet une exploration méthodique et tous azimuts de la couche didascalique proprement dite (je l'appellerai, comme le font bien d'autres, didascalie externe - DEX). Ainsi, au lieu de fournir d'autres preuves de la polymorphie et de la plurifonctionnalité de la DEX, j'ai résolu cette fois de me pencher sur la paradidascalie, c'est-à-dire sur ce qui appartient en droit à la didascalie et qui ne fait pas partie pour autant de la zone textuelle qui lui est normalement réservée. Cette intervention sera donc axée sur le dialogue théâtral qui, plus souvent qu'on ne le pense et de très diverses manières, se fait didascalie ${ }^{3}$. Les renvois systématiques aux textes historiquement un peu rétro - mais dramaturgiquement très avancés - sont là pour prouver que le procédé n'est point chasse gardée des dramaturgies les plus jeunes et des esthétiques subversives: il est vieux comme le théâtre.

\subsection{Narrant, para-narrant et macro-narrant}

Sur le plan énonciatif, la PDI est habituellement assumée soit par des instances conçues exprès à cet effet par le dramaturge, soit par des personnages ordinaires ou quasiordinaires. Quant aux premiers, je les avais nommés narrants pour les opposer 
taxinomiquement aux narrateurs épiques. Les seconds ont reçu l'appellation de paranarrants, vue leur activité narratrice moins intense ${ }^{4}$.

Quoique la narration soit une technique somme toute hétérodoxe au théâtre, on est surpris aussi bien par le nombre de narrants présents dans les textes dramatiques que par la variété de fonctions et de formes qu'ils affectent. Voici un bref relevé de quelques-unes de ces figures : annoncier (Claudel), chœur (Billetdoux, Césaire, Claudel, Ghéon, Shakespeare, etc.), explicateur (Claudel), lecteur (Beckett, Tardieu), meneur de jeu (Ghéon), montreur (Chédid), narrant (Gabily), phono (Cocteau), présentateur (Billetdoux), présentateur-commentateur (Césaire), récitant (Beckett, Césaire, Char, Supervielle), récitante (Césaire), récitants (Claudel), reporter (Billetdoux), rumour (Shakespeare), souvenant (Beckett), speaker (Billetdoux, Césaire).

8 Le narrant et ses satellites, sujets de la narration théâtrale médiatrice (NTM) ${ }^{5}$ ou de la narration paradidascalique (NPD) se définissent par quelques fonctions qui leurs sont traditionnellement dévolues: $1:$ la présentation, l'introduction et l'explication de l'histoire représentée dans la pièce ; 2 : la détermination du cadre circonstanciel des séquences successives et la description des personnages ; $3:$ la prise en charge de toutes sortes de narrations et récits (surtout ceux qui prennent la forme d'une relation simultanée des actions effectuées sur la scène par le sujet lui-même ou par d'autres personnages); 4 :l'organisation $\mathrm{du}$ jeu, définition du cadre participationnel, ordonnancement du spectacle. Le caractère fondamental des narrants et para-narrants tient au fait qu'ils exercent les fonctions esquissées cidessus de manière permanente ou au moins plus d'une fois (pour les paranarrants), créant ainsi une espèce d'armature médiatrice du texte/représentation. Tel est le cas des pièces comme L'Eden Cinéma de Duras, Et les chiens se taisaient de Césaire, Le jeu des merveilles de saint Martin de Ghéon ou encore Has been bird de Billetdoux où toute une équipe de personnages (un véritable macro-narrant) coopèrent à fournir des renseignements didascaliques, récits, images poétiques et commentaires.

9 Afin de mieux délimiter le concept de paradidascalie, il importe d'abord d'exclure certaines variantes du récit teichoscopique à travers lequel sont évoqués des faits passés ou futurs par rapport au zéro déictique de la pièce. Les seules variantes qui méritent l'étiquette de NPD sont celles où les personnages en scène relatent, en simultanéité et le plus souvent au présent, ce qu'eux-mêmes ou d'autres sont en train de faire. Ensuite, il faut mettre hors propos la didascalie interne rigoureusement motivée du point de vue dramaturgique et pragmatique qui ne saurait être perçue comme relevant d'un brouillage modal.

\subsection{Une typologie schématique}

10 Le secteur de la PDI coïncide essentiellement avec deux grands subdomaines de la narration théâtrale. La partie marquée en gris du tableau auquel j'ai abouti en 2007b visualise les 32 types et variantes de la PDI théoriquement repérables ou effectivement repérés à travers le corpus examiné :

\begin{tabular}{|l|l|lr|l|}
\hline $\begin{array}{l}\text { Récit / narration } \\
\text { endodiégétique }\end{array}$ & $\begin{array}{l}\text { Narration } \\
\text { paradidascalique }\end{array}$ & $\begin{array}{l}\text { Narration } \\
\text { médiatrice }\end{array}$ & théâtrale & $\begin{array}{l}\text { Narration } \\
\text { didascalique }\end{array}$ \\
\hline
\end{tabular}




\begin{tabular}{|c|c|c|c|c|c|c|c|c|c|c|c|c|c|c|c|c|c|c|c|c|c|c|}
\hline \multicolumn{2}{|c|}{ Mono } & \multicolumn{3}{|c|}{ Poly } & \multicolumn{3}{|c|}{ Mono } & \multicolumn{3}{|c|}{ Poly } & \multicolumn{4}{|c|}{ Mono } & \multicolumn{4}{|c|}{ Poly } & \multicolumn{2}{|c|}{ Indicative } & \multicolumn{2}{|c|}{ Narrative } \\
\hline S & A & S & & A & end & & exo & enc & & exo & ex & & in & & ex & & In & & pro & dieg & pro & dieg \\
\hline \begin{tabular}{|l|l|l} 
I & E
\end{tabular} & $\mathrm{I}$ & EI & E & I $\mathrm{E}$ & A & $\mathrm{R}$ & \begin{tabular}{l|l}
$A$ & $R$
\end{tabular} & A & $\mathrm{R}$ & $\mathrm{A} / \mathrm{R}$ & $\mathrm{O}$ & I & 0 & I & 0 & I & 0 & I & AR & SR & AR & SR \\
\hline
\end{tabular}

\begin{tabular}{|c|c|}
\hline $\begin{array}{l}\text { Colonne } \\
1:\end{array}$ & $\begin{array}{l}\text { mono }=\text { monologal } ; \text { poly }=\text { polylogal } ; \mathrm{S}=\text { simple (non autonyme) } ; \mathrm{A}=\text { autonyme } ; \mathrm{I}= \\
\text { intrascénique } \mathrm{E}=\text { extrascénique }\end{array}$ \\
\hline $\begin{array}{l}\text { Colonne } \\
2:\end{array}$ & $\begin{array}{l}\text { mono }=\text { monologal } ; \text { poly }=\text { polylogal } ; \text { endo = concernant le personnage-locuteur } ; \text { exo = } \\
\text { concernant d'autres personnages ; } A=\text { autonome (étant la seule source d'information sur } \\
\text { l'univers représenté) } ; \mathrm{R}=\text { redondant (répétant les informations fournies par la DEX ) ; }\end{array}$ \\
\hline $\begin{array}{l}\text { Colonne } \\
3:\end{array}$ & $\begin{array}{l}\text { mono }=\text { monologal } ; \text { poly = polylogal } ; \text { ex = extradiégétique (effectuée par un pesonnage } \\
\text { extérieur à l'action) ; in = intradiégétique (effectuée par un narrant entretenant quelque } \\
\text { relation avec les personnages de la fiction); } \mathrm{O}=\text { off (effectué par une voice off) ; I = in } \\
\text { (effectuée par un narrateur-personnage visualisé : in screen): }\end{array}$ \\
\hline $\begin{array}{l}\text { Colonne } \\
4:\end{array}$ & $\begin{array}{l}\text { indicative = neutre, qui ne fait pas apparaître une subjectivité narratoriale ; narrative = } \\
\text { remplissant des fonctions propres à la narration romanesque } ; \text { pro = proscénique } \\
\text { (relative à un espace scénique inscrit dans le texte) } ; \text { dieg = diégétique (relative à } \\
\text { l'univers représenté) } ; \mathrm{AR}=\text { avec répliques didascalisés } \mathrm{SR}=\text { sans répliques didascalisées. }\end{array}$ \\
\hline
\end{tabular}

Puisque la place est comme toujours rationnée et qu'il serait peu fructueux de reprendre les procédures de naguère, je proposerai ici une grille un peu différente, et, pour ne pas me retrancher derrière des schématisations rassurantes, je discuterai aussi quelques exemples qui posent problème et qui font légion dans les textes.

\begin{tabular}{|l|l|}
\hline \multicolumn{2}{|l|}{ PARADIDASCALIE (PDI) } \\
\hline Type & Fonction \\
\hline $\begin{array}{l}\text { Métathéâtrale } \\
\text { Métadiégétique }\end{array}$ & $\begin{array}{l}\text { exprimer des opinions sur l'art théâtral en général, sur l'ensemble de } \\
\text { l'histoire jouée, introduire et commenter la diégèse ; }\end{array}$ \\
\hline $\begin{array}{l}\text { Diégétique } \\
\text { \& Narration / récit } \\
\text { paradidascaliques (exo- / } \\
\text { endo-) }\end{array}$ & $\begin{array}{l}\text { concernant le monde représenté ; narration/récit paradidascalique } \\
\text { (relation des actions actuellement en devenir); }\end{array}$ \\
\hline Proscénique & $\begin{array}{l}\text { décrire le dispositif scénique, définir le fonctionnement sémantique des } \\
\text { praticables et des accessoires, annoncer les }\end{array}$ \\
\hline & $\begin{array}{l}\text { entrées et les sorties des personnages, commander des changements à } \\
\text { vue, etc. }\end{array}$ \\
\hline
\end{tabular}




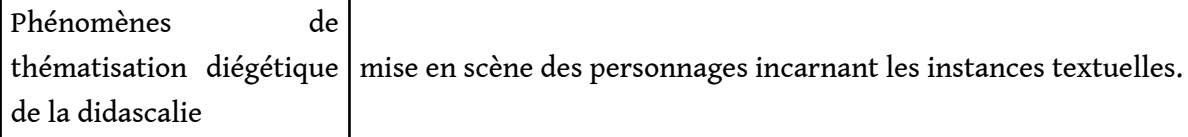

\subsubsection{Paradidascalie métathéâtrale et métadiégétique : « lever les vannes de I'histoire ${ }^{6}$}

Les énoncés didascaliques ont souvent, sinon toujours, une méta-dimension. Ceci est donc également une qualité propre à la PDI. Voici, à titre d'exemple, un fragment du monologue ouvrant la Merveilleuse histoire... de Ghéon (j'y souligne en gras les éléments immédiatement didascalisables, c'està-dire transposables en DEX, et je mets en italiques les énoncés relevant du méta-niveau) :

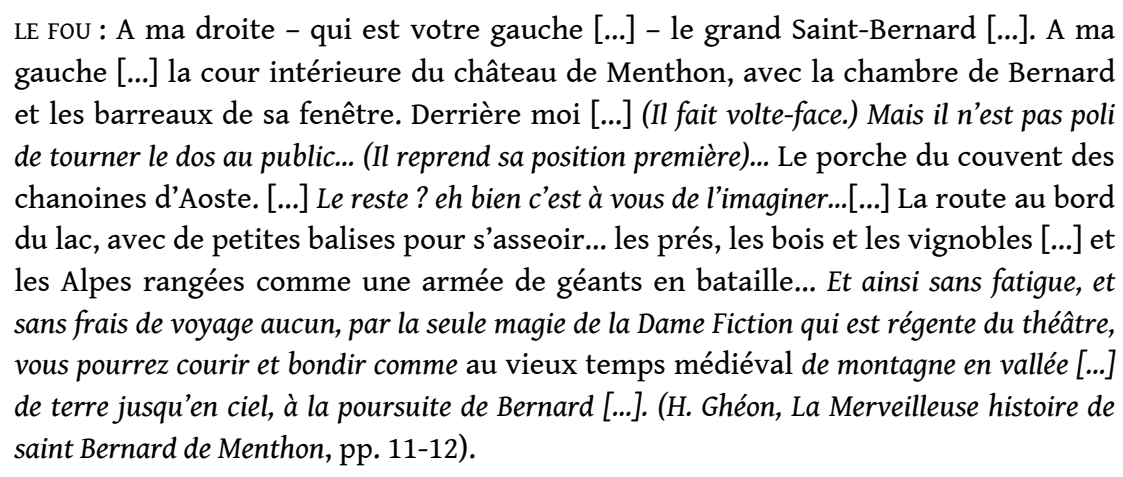

Voilà le décor scénique bien fixé (régime proscénique), le public incité à "imaginer " certaines parties non représentées du monde fictionnel (régime diégétique) et instruit sur le caractère du spectacle (régime métadiégétique), tout ceci étant l'œuvre d'un personnage intégré structurellement au monde fictionnel dans la mesure où il engage des échanges dialogaux avec d'autres personnages. Quant au méta-niveau, le monologue du Fou est plus que riche (je regrette de ne pas pouvoir le citer in extenso...). Alors : 1 : il s'adresse au public: "Mesdames, Messieurs et les autres... », (p. 9) ; 2 : il donne aux spectateurs des instructions sur le mode de réception à adopter «écoutez avec l'oreille de l'esprit»(p.9); 3 : il exprime une opinion sur la plausibilité de l'histoire représentée : «dont l'apparence est fort loin de la vraisemblance »(p. 10); 4 : il définit la structure spatiale de l'univers représenté («bondir [...] de terre jusqu'en ciel »); 5 : il définit la finalité et l'effet perlocutoire escompté du spectacle qui est conçu " afin d'édifier les âmes [...] afin de consterner les dames » (p. 14); 6 : il justifie la mixité générique de l'œuvre: passant du vers à la prose, il affirme qu'il s'agit par là «d'accoutumer» (p. 11) le spectateur à ce balancement ; $7:$ il fait éloge des possibilités de la fiction artistique et en souligne la préséance dans le domaine théâtral. Face à de telles interventions, on est d'ailleurs en droit de poser une question essentielle: jusqu'où s'étend la PDI? Est-on fondé d'envisager comme (para)didascalique tout métacommentaire placé dans la bouche de n'importe quel personnage? Notons aussi en marge une constante qui devra être un jour étudiée en détail : la cohabitation de la DEX et de la PDI. 


\subsubsection{Paradidascalie diégétique et narration paradidascalique : « on voit ce qui est dit ${ }^{7}$}

15 La PDI diégétique est celle qui contribue à la construction de l'univers fictionnel sans faire allusion à l'espect technique de la représentation. Pour voir un cas flagrant, ouvrons Le Livre de Cristophe Colomb. L'aventure du grand explorateur y est censée émaner de la lecture du Livre faite par l'Explicateur'. C'est de la manière suivante que celui-ci plante le décor et présente le protagoniste :

L'EXPLICATEUR. - Une pauvre auberge à Valladolid. (En effet, on voit sur la scène une pauvre auberge à Valladolid). Christophe Colomb! (Entre Christophe Colomb.) Il est vieux. Il est pauvre. Il est malade. Il va mourir bientôt. L'homme qui est avec lui et qui discute est l'aubergiste. Le vieillard a le licou de sa mule autour du bras. C'est son seul bien, il a peur qu'on la lui vole. (P. Claudel, Le Livre de Christophe Colomb, p. 1142-1143)

16 Ni didascalie, ni dialogue - encore que, topologiquement, nous soyons bien dans la zone des répliques - ce monodiscours monstratif convoque un personnage, en crayonne un portrait, en rapporte les sentiments, en signale un acte de parole et en prédit le proche avenir. Monodiscours entièrement didascalisable, du reste - pas besoin de tenter l'épreuve de la transmodalisation: il suffirait d'enlever le point d'exclamation après «Colomb» et le texte tournerait à la DEX. Pour pouvoir parler d'une NPD idéale, il faudrait encore que l'action prime un peu davantage la description, mais déjà tel quel notre exemple illustre assez bien le prinicpe de la relation simultanée et du redoublement codique $^{9}$ propre à la NPD. Et s'il existe parfois "des formes de similitude ou de continuité entre le dialogue et les didascalies » (Jolly 2007 : 71), l'extrait qu'on vient de lire en fournit une bonne preuve : la NPD et la DEX sont ici bel et bien reliées par l'articulateur en effet grâce auquel on passe de l'une à l'autre avec une parfaite fluidité. Tout se passe comme si le didascale entendait la voix du narrant et enchaînait sur elle comme si de rien n'était. La DEX et la PDI, syntaxiquement soudées, font ici en plus double emploi, puisque la DEX n'ajoute rien qui vaille du point de vue sémantique. Ce phénomène de synchronisation des codes (audio-visuel et discursif) ou du double encodage est d'ailleurs plus fréquent ${ }^{10}$ qu'on ne l'imagine, les auteurs tenant justement à ce que le dit jouxte le vu, si l'on peut dire ainsi. Voici un autre exemple où la NPD revêt une forme clairement identifiable :

L'Ange Gardien, Dona Prouhèze.

Le ravin profond qui entoure l'auberge, plein de ronces, de lianes et d'arbustes entremêlés. Sur le bord se tient l'Ange Gardien en costume de l'époque et l'épée au côté.

L'ANGE GARDIEN. - Regardez-là qui se démène au milieu des épines et des lianes entremêlées, glissant, rampant, se rattrapant, des ongles et des genoux, essayant de gravir cette pente abrupte ! et ce qu'il y a dans ce cœur désespéré ! / Qui prétend que les Anges ne peuvent pas pleurer ? [...]

[...] Dona Prouhèze sort du fossé. Elle est en vêtements d'homme, tout déchirés, les mains et la figure meurtries. Elle se rajuste tant bien que mal, inspecte les alentours.

(P. Claudel, Le Soulier de satin, Ière journée, s. XII)

Notons, à travers ce passage, la parfaite complémentarité opérative de la couche didascalique et paradidascalique : la DEX dessine le décor et introduit le personnage d'Ange ; celui-ci prend en charge, à l'aide de la NPD, le segment se déroulant dans le fossé et laisse à la DEX le soin de conclure. Mis à part quelques éléments réfractaires à la didascalisation (tournures injonctives, notations sur les états d'âme et marques de 
subjectivité que l'on pourrait tout de même didascaliser au prix de petites pertes en signifiance), la réplique de l'Ange est aisément transposable en DEX.

Par contre, avec l'extrait suivant, la didascalisation serait bien plus délicate à opérer. Il s'agit d'un extrait du Tableau II de La Mort au Donateur de Patrice de la Tour du Pin. Conformément au principe d'adialogisme qui caractérise les dispositifs énonciatifs paradidascaliques, on n'a ici qu'un pseudo-échange :

Une prairie. Les enfants, puis la voix de l'homme.

LES ENFANTS PARADISIERS / Qui va par la prairie / Et balaye la lune? / C'est un mauvais plaisant / À la recherche d'une / Plaisanterie...

LES ENFANTS SAUVAGES / Aucun de vous ne semble / La trouver à son goût. / Nous ne voyons personne, / Mais sous les pas d'un fou, / Les herbes tremblent...

LES ENFANTS CHANTEURS / Quelques enfants chanteurs / Sont alors survenus, / Ils ont caché la lune, / Et l'autre s'est perdu, / Perdu ailleurs...

LA VOIX DE L'HOMME (à part) / C'est bien; le premier pas est fait, ils sont en attente, sentent bien que quelque chose a peur en eux. Ils ne dansent plus, se serrent les uns contre les autres; ce n'est pas leur langage que je parle, ils ne peuvent pas m'entendre, mais ils tendent le cou... (Patrice de la Tour du Pin, La Mort au Donateur, p. 97)

Mi-poème, mi-parabole, le texte de la Tour du Pin a un format extérieur d'un texte dramatique, mais les migrations illicites du didascalique vers le dialogal sont perceptibles à l'œil nu. Dans le passage ci-dessus relevons 1 : la relation in vivo, d'allure paradidascalique, que font les Enfants Paradisiers et les Enfants Sauvages de l'arrivée d'un individu invisible ; 2 : la réplique aux contours vaguement paradidascaliques des Enfants Chanteurs qui parlent d'eux-mêmes en employant la troisième personne grammaticale (selon les interprétations qu'on pourrait en donner, il s'agirait soit d'un mini-segment d'endo-NPD, soit d'un récit de suppléance) ; 3 : les paroles de l'Homme qui se teignent elles aussi de coloris paradidascalique, en nous faisant connaître les actions et les sentiments des Enfants que nous voyons en même temps évoluer sur le plateau. Tout cela, comme je l'ai dit, est quelque peu flou, et montre encore une fois qu'il est difficile de faire le départ entre les segments paradidascaliques (c'est-à-dire didascalisables) implantés dans le dialogue et d'autres types d'énoncés qui n'altèrent en rien l'homogénéité générique du message théâtral.

\subsubsection{Paradidascalie proscénique : « on a parfaitement bien représenté ici... »}

La PDI proscénique, comme son nom l'indique, se réfère à l'espace concret d'un plateau virtuel. Dans l'extrait ci-dessous, l'Annoncier s'adresse à un public fictif réuni dans une salle imaginaire, oriente le regard des spectateurs vers un point concret de la scène et utilise un métalangage théâtral :

L'ANNONCIER. [...] - Fixons, je vous prie, mes frères, les yeux sur ce point de l'océan Atlantique [...] à égale distance de l'Ancien et du Nouveau Continent. On a parfaitement bien représenté ici l'épave d'un navire démâté qui flotte au gré des courants. [...] (P. Claudel, Le Soulier de satin, pp. 942-943)

21 Ces deux énoncés exemplifieraient ainsi une pure PDI proscénique, mais peut-on réellement dissocier l'ordre proscénique et diégétique ? C'est douteux. La double (voire la triple) référentialité du signe (dans le texte) théâtral renvoyant à la fois à une scène virtuelle, à une fiction diégétique et au monde empirique, fait que les ordres proscénique, diégétique et métadiégétique/ métathéâtral se trouvent sinon inextricablement mêlés, du moins coprésents à travers une mosaïque de segments 
susceptible d'une analyse séquentielle à la J.M. Adam... A la manière claudelienne, la DEX ajoute du reste juste après : «

On distingue du reste suffisamment ce que décrit l'Annoncier» (p.942). Cette concurrence ou co-occurence du signe visuel et du signe discursif que l'auteur tient à maintenir est un choix artistique: aussi contraire que ce soit à l'économie de la représentation, l'intention auctoriale est que la parole seconde l'image et que l'une signifie conjointement avec l'autre (sans le commentaire verbal de la PDI diégétique, le spectateur saurait-il que la scène se trouve "à distance égale » entre l'Amérique et l'Europe ? Plutôt non).

\subsubsection{Paradidascalie sous la forme de la didascalie personnifiée}

Le dernier de nos exemples est une curiosité, une limite théorique, mais il illustre la quatrième case de notre tableau récapitulatif (phénomènes de thématisation), et c'est à ce titre que je tiens à en parler brièvement. L'exemple provient d'une pièce peu connue de Claudel, La Lune à la recherche d'elle-même, pièce destinée à la radio, ce qui explique en partie le recours au procédé que nous allons examiner. On voit en effet dans La Lune... (sous-titrée «Extravaganza radiophonique») un curieux personnage appellé Le Préposé aux Indications Scéniques, alias Prépo, agent explicitement chargé de dire la didascalie sur la scène et de bien plus que cela... Voilà comment il s'acquitte de sa mission :

LE CH৫UR. - [...] Et moi, si voulez bien, je bois un coup. À votre santé.

LE PREPOSE AUX INDICATIONS SCENIQUES. - Il boit un coup.

(P. Claudel, La Lune..., p. 1280)

24 Après ce redoublement de l'information auquel nous nous sommes déjà habitués, voici que, tout à coup, un autre redoublement (ou dédoublement) se produit, bien plus intéressant :

volPILLA. - Fiche le camp, on t'a assez vu! Quel bavard!

LE PREPO NUMERO 1. - Il fiche le camp, on l'a assez vu.

LE PREPO NUMERo 2. - Et La Police faunesque amène le Poëte devant le Tribunal,

composé des deux personnages décrits. (P. Claudel, La Lune..., p. 1283)

La boucle est ainsi bouclée: si le dialogue se fait parfois didascalie, ici la didascalie s'incarne dans un personnage qui se scinde en deux de manière à mettre en place un simulacre de dialogue. Cette technique a des implications génériques de deux types: 1 : par le biais du dédoublement du sujet de la PDI, le dramaturge obtient une théâtralisation artificielle d'un procédé narratif; $2:$ il pratique une écriture didascalique quelque peu caricaturale vis-à-vis d'elle-même ( Il fiche le camp, on l'a assez vu »). Il est clair par ailleurs qu'une telle manière de procéder est dictée par les contraintes de la diffusion radiophonique pour laquelle le texte a été conçu.

Agent actif à part entière, le Prépo n'hésite pas à s'avancer en dehors du champ opératoire que son nom lui assigne, de sorte que sa présence est perçue comme une perturbation par d'autres personnages qui protestent :

volPILLA. - Tout ça n'est pas pour Strombô. Alors a fait ce qu'elle peut, c'te pauv'e

créature.

UNE VOIX QUELQUE PART. - L'œil n'est rien, c'est le regard.

DANSE LA NUIT. - Pardon, Monsieur le Prépo, vous êtes ici pour indiquer les indications et non pas pour nous faire part de vos idées personnelles. 
LE PREPO. - Je n'ai rien dit.

(P. Claudel, La Lune..., p. 1287) perfectibles. L'essentiel est de saisir la spécificité de la médiation théâtrale, puisque le théâtre fait de plus en plus souvent appel aux techniques de médiation narrative ou poétique. Dans l'optique strictement didascalique, on doit en tout cas insister sur le rapport entre la parole de narrant, la DEX traditionnelle. La possibilité ou l'impossibilité de la transposition didascalique est en effet l'un des critères majeurs d'analyse du discours du narrant et une donnée importante pour la détermination de son degré de motivation. Si le discours du narrant est aisément transposable en DEX, son usage risque d'apparaître comme quelque peu forcé. Et, inversement, la difficulté à traduire le langage du narrant en langage didascalique militerait en faveur de l'utilité (toujours relative) de ce type de personnage.

31 La PDI soulève également d'importantes questions du point de vue scénocentriste. En effet, l'un des problèmes majeurs de la PDI est qu'elle double tantôt partiellement, tantôt presque complètement, la composante audiovisuelle du spectacle. Pour le 
lecteur et l'éventuel metteur en scène, la PDI, conjuguée avec la DEX (si présente), reste d'habitude l'unique source d'information sur l'aspect matériel du spectacle. Il n'en va pas de même pour le spectateur d'une création scénique concrète, lequel perçoit un message parallèle. Quels sont les effets sémantiques produits par un tel parallélisme? Les capacités cognitives des spectateurs et la perplexité des metteurs en scène qui déclarent forfait devant certains textes ne seraient-elles pas elles aussi à prendre en compte comme facteurs déclencheurs de certaines pratiques scripturales? Des figures audio-visuelles, dont le langage est souvent imprécis et mal codé en général, ne sontelles pas plus faciles à lire si elles s'accompagnent de discours explicatif?

On peut s'interroger finalement sur le pourquoi du phénomène en posant des questions suivantes: s'agit-il d'une technique visant tout simplement à diversifier les moyens d'expression théâtrale ou à en dynamiter les conventions? L'invasion de la DEX et l'inflation respective de la PDI témoignent-t-elles de la volonté ferme des auteurs d'être (com)pris et représentés à la lettre et comme ils l'entendent ? Est-on, avec la PDI (et la didascalie énoncée sur la scène) en face d'une revalorisation plus profonde des rapports entre le polysystème théâtral et le langage naturel?

\section{BIBLIOGRAPHIE}

Basile [Sandrine], 2007, « ...Jean-Luc Lagarce ou la didascalie comme principe d'écriture intime », in : La Didascalie dans le théâtre du XXe siècle, Dijon, EUD, pp. 101-111.

Bernanonce [Marie], 2007, « Pour une typologie de la vois didascalique... », in : La Didascalie dans le théâtre du XXe siècle, Dijon, EUD, pp. 47-60.

Châteauvert [ Jean ], 1993, « Narrer ou ne pas narrer. Pour repenser la relation d'attribution narrative », in : Poétique, 93, pp. 91-111.

Fix [Florence], Toudoire-Surlapierre [Frédérique], 2007, La Didascalie dans le théâtre du XXee siècle, Dijon, EUD.

Gaudreault [André], 1999, Du littéraire au filmique. Système du récit, Paris, Armand Colin \& Nota Bene.

Golopentia [Sanda], 1993, « Les didascalies de la source locutoire », in : Poétique, 96, pp. 475-492.

Ingarden [Roman], 1931, Das literarische Kunstwerk, Halle (Saale), Max Niemeyer Verlag.

Issacharoff [Michael], 1985, Le Spectacle du discours, Paris, José Corti.

Jolly [Geneviève], 2007, «L'importance des voix didascaliques dans Purifiés de Sarah Kane », in : La Didascalie dans le théâtre du XXe siècle, Dijon, EUD, pp. 61-74.

Klinkenberg [Jean-Marie], 2002 (1996), Précis de sémiotique général, Paris, De Boeck, « Points / Essais ».

Martinez Thomas [Monique] et Golopentia [Sanda], 1994, Voir les didascalies, Toulouse, CRIC \& ORPHYS. 
Mouratidou [Eleni], 2007, « De l'indication scénique à l'acte dramatique... A propos des didascalies narrées... », in : La Didascalie dans le théâtre du XXe siècle, Dijon, EUD, pp. 75-86. Pruner [Michel], 2001, Analyse du texte de théâtre, Paris, Nathan/HER.

Thomasseau [Jean-Marie], 1998, « Le récit paratextuel », in : Le Récit et les arts, Paris, l'Harmattan. Wołowski [Witold], 2007a, « Sur quelques formes de l'écriture didascalique », in : La Didascalie dans le théâtre du XX $X^{e}$ siècle, Dijon, EUD, pp. 21-33.

Wołowski [Witold], 2007b, Du Texte dramatique au texte narratif. Procédés interférentiels et formes hybrides dans le théâtre français du XX $X^{e}$ siècle, Lublin,

Wydawnictwo KUL, 2007.

Wołowski [Witold], 2007c, « Lecture à haute voix dans le texte dramatique », in : Lubelskie Materiały Neofilologiczne, Lublin, Uniwersytet Marii-Curie Skłodowskiej, nº31, ss. 51-67.

Zaragoza [Georges], 2007, « La didascalie poétique... », in : La Didascalie dans le théâtre du XXe siècle. Regarder l'impossible, Dijon, EUD, pp. 61-74.

\section{Textes évoqués ou cités}

Billetdoux [François], Has been bird, ronéotype non publié, 1964.

Césaire [Aimé], Et les chiens se taisaient, Paris, Gallimard, 1970.

Claudel [Paul], Le Soulier de satin, in : Théâtre II, Paris, Gallimard, « Pléiade », 1956.

Claudel [Paul], Le Livre de Christophe Colomb, in : Théâtre II, Paris, Gallimard, « Pléiade », 1956.

Claudel [Paul], La Lune à la recherche d'elle-même, in : Théâtre II, Paris, Gallimard, « Pléiade », 1956.

Cocteau [Jean], Les Mariés de la Tour Eiffel, Paris, Gallimard, 1978.

Duras [Marguerite], L’Eden Cinéma, Paris, Mercure de France, « Folio », 1989 (1977).

Eschyle, Les Perses, in : Les Tragiques grecs. Eschyle, Sophocle, Euripide. Théâtre complet, Editions de Falloi, 1999.

Ghéon [Henri], Le Jeu des merveilles de Saint Martin improvisé par les anges, Paris, André Blot Editeur, «Les cahiers du théâtre chrétien », 1942.

Ghéon [Henri], La Merveilleuse histoire du jeune Bernard de Menthon, Paris, André Blot Editeur, 1942.

La Tour du Pin [Patrice], La Mort au Donateur, in : Une Somme de poésie, Paris, Gallimard, 1946.

\section{NOTES}

1. Didascalie de la source locutroire (Golopentia 1993). En outre, j'emplois ici quelques autres sigles pour remplacer les termes clefs de l'article: DEX - didascalie externe; PDI paradidascalie ; NPD - narration / récit paradidascaliques.

2. La distinction ingardenienne entre texte principal et texte secondaire (Haupttext vs Nebentext) remonte aux années 1930 et non pas, comme le croient et répètent la plupart des théâtrologues occidentaux, à ses travaux en langue polonaise des années 1950 traduits en français à partir du début des années 1970 et même 1980 pour son opus magnum. De la sorte, on retarde injustement de 40 , voire de 50 ans, les remarques du philosophe. 
3. Ces contaminations du dialogue par des éléments didascaliques ont donné lieu aux désignations telles que didascalies textuelles (ou implicites) (Pruner 2001 :17) ou dialogue didascalique (Bazile 2007 : 108).

4. Il serait également possible d'adopter ici le terme abstrait de didascale forgé par $\mathrm{M}$. MartinezThomas (1994), à condition toutefois de lui faire subir une extension importante, c'est-àdire l'étendre sur les personnages concrets. De façon ou d'autre les narrants, considérés dans leurs mission d'oralisateurs des paradidascalies, ne sont en fait qu'une in c a r n a t i o $\mathrm{n}$ du didascale tel que le conçoit la théoricienne toulousaine.

5. La figure du narrant et toutes les figures médiatrices au théâtre devraient être mises en relation avec les différents types de bonimenteur, narrateur filmique montré. Il est passionnant de lire à ce sujet l'excellente étude de J. Châteauvert (1993) et surtout le monumental traité de la narratologie littéraire, théâtrale et filmique d'A. Gauderault (1999). Dans le contexte plus strictement dramaturgique, le «côté didascale» du narrant se manifeste souvent dans les diverses formes d'encadrement prologal-épilogal, question que j'aborde plus en détail en $2007 b$. Enfin, nous sommes ici à la jonction des deux phénomènes proches parents: PDI et didascalie prononcée sur la scène. Je ne suis pas, pour ma part, enthousiaste de l'étiquette didascalie narrée proposée par E. Mouratidou (2007), car le terme narré me semble référer à un autre ordre de phénomènes. Par contre tous les problèmes de coprésence des codes linguistique et audio-visuel que Mouratidou envisage dans son article me semblent effectivement de première importance pour tout le secteur des arts du spectacle.

6. La citation est empruntée au discours paradidascalique de l'Annoncier claudelien qui définit ainsi son rôle de narrant (Le Soulier de satin, p. 1042).

7. C'est une brève DEX tirée de L'Eden Cinéma de Duras (p. 32), laquelle double les paroles de Suzanne.

8. La paradidascalie fait parfois objet de lecture, l'oralisateur paradidascalique étant même dans certains cas présent corporellement sur la scène le livre à la main. Pour l'analyse de quelques cas de figure $c f$. W. Wołowski (2007c).

9. Il n'est double (concrètement) qu'à la représentation, à moins que la DEX répète en écho le contenu de la NPD.

10. Les dramaturges évitent les reprises mot à mot dont la redondance saute aux yeux. Il est plus fréquent qu'une DEX a un contenu comparable. Ainsi chez Cocteau : Phono Un : «Funérailles du général » (PDI) et la DEX ajoute : «Cortège funèbre » (Les Mariés..., p. 62). 